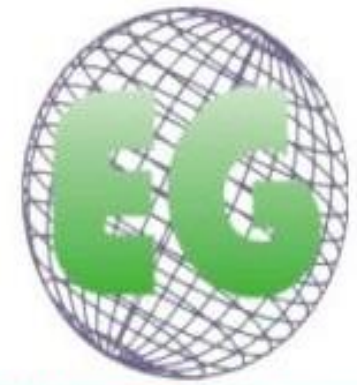

ISSN 1695-6141 $N^{\circ} 45$

\title{
Valoración del dolor en pacientes intervenidos quirúrgicamente de hernioplastia con cirugía ambulatoria en el Hospital Universitario Santa María del Rosell
}

Evaluation of pain in surgically operated patients with hernioplasty with outpatient surgery at the University Hospital Santa Maria del Rosell

\section{*Asuar López, María Ángeles}

\author{
*Enfermera. E-mail: geles_187@hotmail.com
}

\author{
http://dx.doi.org/10.6018/eglobal.16.1.228981
}

\section{RESUMEN}

Introducción/Objetivos: Se realiza un estudio para valorar la calidad del manejo del dolor hospitalario y extrahospitalario durante las primera 24 horas con la Escala Analógica Visual y Verbal del dolor (EVA) en cirugía mayor ambulatoria (CMA) de los pacientes intervenidos de hernioplastia, conocer los analgésicos más utilizados y confirmar que tienen un adecuado control del dolor (EVA $\leq 3$ ).

Material y Métodos: Estudio observacional, longitudinal y descriptivo para valorar el Dolor Agudo Postquirúrgico (DAP) y los analgésicos precisos para controlar el dolor; desarrollado en el Servicio de Anestesia y Reanimación del Hospital Universitario Santa Maㅡ del Rosell del Área II de Cartagena durante los meses de octubre a diciembre de 2014. Los participantes fueron individuos mayores de edad intervenidos quirúrgicamente de hernioplastia con riesgo anestésico según el sistema de clasificación American Society of Anesthesiologists (ASA) I-II, y III muy seleccionados. El análisis estadístico se realizó con el paquete estadístico SPSS para Windows versión 19.0.

Resultados: Participaron 70 individuos. Refirieron EVA $\leq 1$ un $80 \%$ al ingreso en reanimación, un 98,6\% al alta en reanimación, un $82,6 \%$ al alta del CMA y un $87 \%$ en su domicilio. Todos fueron infiltrados con anestésico local y los analgésicos más utilizados fueron los "no opiáceos".

Conclusiones: La mayoría de los individuos durante las primeras 24 horas postquirúrgicas refirieron un EVA $\leq 1$ presentando un adecuado control del dolor, siendo los analgésicos "no opiáceos" los más utilizados junto con las infiltraciones locales.

Palabras clave: dolor agudo postquirúrgico; escala visual analógica del dolor; Cirugía mayor ambulatoria; analgésicos y anestésicos 


\section{ABSTRACT}

Introduction / Objectives: A study was carried out to evaluate the quality of hospital and out-ofhospital pain management during the first 24 hours with the Visual and Verbal Analog Pain Scale (VAS) in major outpatient surgery of patients undergoing hernia repair, Know the most commonly used analgesics and confirm that they have adequate pain control (EVA $\leq 3$ ).

Material and Methods: Observational, longitudinal and descriptive study to assess Acute PostOperative Pain and analgesics to control pain; Developed in the Anesthesia and Resuscitation Service of the Santa Ma del Rosell University Hospital of Area II of Cartagena during the months of October to December of 2014. The participants were elderly individuals surgically operated on hernioplasty with anesthetic risk according to the American classification system Society of Anesthesiologists (ASA) I-II, and III highly selected. Statistical analysis was performed with the SPSS statistical package for Windows version 19.0

Results: 70 individuals participated. They reported EVA $\leq 180 \%$ at resuscitation admission, $98.6 \%$ at resuscitation discharge, $82.6 \%$ at CMA discharge and $87 \%$ at home. All were infiltrated with local anesthetic and the most commonly used analgesics were "non-opiates".

Conclusions: Most individuals during the first 24 postoperative hours reported an EVA $\leq 1$ with adequate pain control, with non-opioid analgesics being the most used together with local infiltrations.

Keywords: Acute postoperative pain; Visual analogue scale of pain; Major outpatient surgery; Analgesics and anesthetics.

\section{INTRODUCCIÓN}

La hernia de pared abdominal (HPA) se define como un defecto en la continuidad de las estructuras fasciales y/o músculo-aponeuróticas de la pared abdominal que permiten la salida o protrusión de estructuras que normalmente no pasan a través de ellas. Su localización anatómica más frecuente es la región inguinal o umbilical. Actualmente constituye por su elevada incidencia un problema social, y desde el punto de vista quirúrgico, una de las intervenciones más frecuentemente realizadas a nivel mundial. ${ }^{1-4}$ En su práctica quirúrgica actual por laparotomía, se espera, además de bajas recidivas, una recuperación rápida y segura.

La cirugía mayor ambulatoria (CMA) ha experimentado un desarrollo considerable desde los años 60 y se define como aquella en la cual el paciente es dado de alta el mismo día de la intervención después de un tiempo variable en observación, sin hospitalización. ${ }^{5}$ La hernioplastia en CMA se realiza de dos formas, mediante anestesia local junto a una sedación, y asociada a un alta precoz, o empleando anestesia general o espinal y vinculada a una hospitalización retardada (hasta la tarde del día de la intervención). ${ }^{6}$

La elección de la técnica anestésica en hernioplastia está supeditada a la magnitud del problema herniario, lo que lleva aparejado una mayor demora en el procedimiento anestésico, a las ventajas de uno u otro procedimiento quirúrgico y anestésico y su posible efecto indeseado. ${ }^{7}$ En la actualidad, sumado a la mejora de las técnicas quirúrgicas, se está priorizando el concepto de deambulación precoz, hospitalización corta y una mayor preocupación por un postoperatorio sin dolor. ${ }^{7,8}$

Cushing fue el primero en difundir el empleo de la anestesia local para el tratamiento de la hernia inguinal. Las evidencias actuales demuestran ventajas como mejor costo beneficio, menor morbilidad y mayor satisfacción del paciente, ya que éstos no pierden la capacidad de movilización y deambulación por sí mismos, no presentan los efectos colaterales indeseables del bloqueo regional como retención urinaria y/o 
punción de la duramadre. ${ }^{1,}$ 3-4 $\mathrm{Su}$ invasión es menor, ya que produce un efecto selectivo sobre el área en que se realiza la intervención, sin que se interfiera en la inervación de otros órganos.

Esta técnica requiere de la colaboración del paciente y por ello no es recomendable en niños o pacientes con enfermedades psiquiátricas. Otra ventaja de la anestesia local es que no es necesario interrumpir la ingestión de alimentos, ni la administración de fármacos, aspecto a tener en cuenta con los pacientes diabéticos. ${ }^{8}$ Todo ello permite agilizar el alta a domicilio dado que no requiere de mayor cuidado ni asistencia. $^{1,8}$

Las reacciones adversas apreciadas durante la cirugía realizada con anestesia local son: el dolor, el síndrome vasovagal, la inquietud y la hipertensión. El dolor debe ser tratado con la administración controlada de analgésicos durante la intervención. Las crisis vagales, caracterizadas por palidez, sudoración, nauseas, bradicardia e hipotensión se tratan mediante atropina intravenosa. La inquietud hace necesaria el uso de sedantes. La hipertensión requiere por regla general solo una actitud expectante por parte del anestesista y/o cirujano. ${ }^{8}$

La anestesia raquídea es el tipo de anestesia neuroaxial que se obtiene al depositar un anestésico local en el espacio subaracnoideo, de donde se distribuye según sus factores físicos para producir un bloqueo reversible de las raíces nerviosas raquídeas con pérdida temporal de la actividad motora, sensitiva y autónoma. Tiene grandes ventajas, entre las cuales destacan el inicio rápido de la analgesia, y el control de la altura del bloqueo según la dosis y el volumen administrado. A pesar de los múltiples factores que determinan la difusión del anestésico, con la anestesia raquídea se obtienen condiciones muy seguras y satisfactorias. ${ }^{9}$ Dentro de las complicaciones derivadas de la anestesia espinal, es frecuente la hipotensión inducida por la anestesia (por disminución del retorno venoso), la cefalea y la retención urinaria aguda (RAO). ${ }^{3,10,11}$

Se han producido importantes avances en las técnicas de la anestesia general, de modo que se alcanza con creces uno de los principales objetivos de la Cirugía Mayor Ambulatoria como es el de obtener mayor confort perioperatorio para el paciente, minimizando las complicaciones postanestésicas y proporcionando mayor seguridad, de modo que se acorten los tiempos de estancia en las unidades de CMA. El uso de la mascarilla laríngea ha cambiado la práctica anestésica, reemplazando a la intubación endotraqueal, lo cual hace innecesario el uso de relajantes musculares, consiguiendo una recuperación postanestésica más rápida, siendo su uso una técnica alternativa en los pacientes de cirugía ambulatoria. Su seguridad y eficacia ha sido demostrada y su utilización es de elección en la mayoría de las intervenciones. ${ }^{3}$

La International Association for the Study of Pain (IASP) define el dolor como la experiencia sensorial y emocional desagradable producida por una lesión tisular real o potencial que se descubre en términos de dicha lesión. ${ }^{12,13} \mathrm{El}$ dolor agudo postquirúrgico (DAP) es un dolor repentino secundario a una agresión directa o indirecta producida por el acto quirúrgico. ${ }^{12}$ Puede ser también producido por la técnica anestésica, las posturas inadecuadas, contracturas musculares, distensión vesical o intestinal, etc.... ${ }^{14}$ Hasta el momento actual, el DAP persiste como un síntoma de elevada prevalencia, cuya incidencia en España se sitúa entre un 46-53\%, incluyendo a los pacientes sometidos a cirugía ambulatoria, según una encuesta sobre el DAP publicada en 2004 por la Sociedad Española del Dolor (S.E.D.) ${ }^{15}$ 
La intensidad del dolor está influenciada por factores físicos, psíquicos y emocionales, aspectos de la personalidad, factores sociales, culturales e interpersonales, así como por la existencia de una experiencia previa frente al dolor. ${ }^{13}$

Todas las intervenciones quirúrgicas van seguidas de la aparición de dolor, este síntoma va a producir respuestas metabólicas endocrinas, reflejos autonómicos, nauseas, íleo, espasmo muscular y morbimortalidad postoperatoria. ${ }^{13,}{ }^{15}$ Las características e intensidad del dolor postoperatorio son previsibles, por lo que lo ideal es anticiparnos a éste y evitar sus inconvenientes y complicaciones. El abordaje del tratamiento del DAP requiere un abordaje multidisciplinario, en el cual, el personal de enfermería tiene un papel vital en el control del dolor agudo postquirúrgico. ${ }^{12,16}$

Para un efectivo control del dolor, es necesario un abordaje multimodal, con la utilización de fármacos con distintos mecanismos de acción, consiguiéndose un efecto aditivo y menos efectos adversos. ${ }^{17} \mathrm{Al}$ iniciar este abordaje antes del acto quirúrgico, estamos dando analgesia anticipada y preventiva. ${ }^{14,} 18$ Para las asociaciones analgésicas se utilizan tres grupos de fármacos analgésicos según la escala analgésica de la O.M.S: el grupo de los no opiáceos que incluye a los antiinflamatorios no esteroideos (AINES), al paracetamol y al metamizol; los opiáceos menores o débiles como la codeína y el tramadol; y los opiáceos mayores o potentes como la morfina. ${ }^{12,15,19}$

Los opioides han sido la base del tratamiento del dolor postoperatorio, debido a su potencia, se utilizan para el manejo del dolor moderado y severo. Producen una analgesia de alto grado sin efecto techo, pero sus efectos adversos como la depresión respiratoria, sedación, trastornos del nivel de conciencia, nauseas y vómitos, hacen que su uso esté limitado, utilizando otros analgésicos no opioides para complementar, y en ocasiones reemplazarlos, con el fin de mejorar la analgesia, facilitando la rehabilitación del paciente tras la intervención. ${ }^{14}$ Entre los principales opioides se encuentra el tramadol, opioide sintético, y su uso está más extendido que el del resto de los opiáceos, ya que tiene menos efectos secundarios que la morfina. ${ }^{20}$

Todos los AINES tienen efectos analgésicos, antiinflamatorios y antipiréticos. Son fármacos que se usan frecuentemente para el manejo del dolor agudo y para los procesos inflamatorios. Tienen una menor potencia que los opiáceos, y se usan a menudo como coadyuvantes de éstos para disminuir sus efectos secundarios. ${ }^{17}$ Sus principales efectos adversos son gastrointestinales: úlcera péptica, renales: disfunción renal, hematológicos, de hipersensibilidad y del sistema nervioso central. ${ }^{13}$

El paracetamol o acetaminofén es un coadyuvante eficaz para la analgesia con opioides, disminuyendo las necesidades de estos últimos o las puntuaciones de dolor en un 20-30\%. Se considera que tiene efectos analgésicos y antipiréticos, pero no se le considera antiinflamatorio. Además, manifiesta un efecto potenciador de los AINES, no presentando efectos adversos relevantes a dosis terapéuticas. ${ }^{14,20}$

La evaluación de la intensidad del DAP es difícil porque no existe un método totalmente preciso. ${ }^{21}$ Para valorar la intensidad del DAP se desarrollaron las escalas de autoevaluación o valoración subjetiva. Como ejemplos se pueden citar la Escala Verbal Numérica (EVN), Escala de expresión facial, Escala verbal simple, la Escala Visual Analógica (EVA) que es actualmente la escala más utilizada como herramienta de referencia para medir el DAP. ${ }^{12}$ La EVA consiste en una línea horizontal o vertical 
de $10 \mathrm{~cm}$ de longitud dispuesto entre dos puntos donde figuran las expresiones "no dolor" y "máximo dolor imaginable" que corresponden a las puntuaciones de 0 y 10 respectivamente; en el cual, el paciente marcará aquel punto de la línea que mejor refleje el dolor que padece. ${ }^{18,19}$ Scott y Huskisson en 1976, fueron los que pensaron en aplicar la Escala Visual Analógica para evaluar el dolor de una manera objetiva. En diversos estudios se ha demostrado que es un instrumento sólido, sensible al cambio, y por lo tanto fiable de usar, demostrándose así su fiabilidad y validez. ${ }^{22}$

Se decidió realizar este estudio para valorar la calidad del manejo del dolor hospitalario y extrahospitalario en cirugía ambulatoria de los pacientes intervenidos de hernioplastia y conocer si tienen un buen control del dolor en su domicilio con el protocolo de analgesia que le da al alta el anestesista encargado de reanimación y la unidad de CMA. Para ello, se hizo una valoración del dolor con la Escala Visual Analógica que llevaba incorporada la Escala Visual Numérica y la Escala de Expresión Facial.

El objetivo principal planteado es:

- Valorar la EVA en pacientes intervenidos de cirugía de pared abdominal (hernia inguinal, ventral, etc.), en el postoperatorio inmediato en el Hospital Universitario Santa $\mathrm{M}^{\mathrm{a}}$ del Rosell y durante las primeras $24 \mathrm{~h}$ en su domicilio.

Nuestros objetivos específicos son:

- Conocer mediante escalas de valoración del dolor, el porcentaje de dolor postoperatorio sentido por los pacientes intervenidos de hernioplastia al ingreso y durante su estancia en reanimación, al alta del CMA y a las $24 \mathrm{~h}$ de la intervención, ya estando en su domicilio.

- Conocer los analgésicos utilizados en el intraoperatorio, en reanimación como rescate ante un pico de dolor o profilaxis, y los administrados en su domicilio.

- Confirmar que los pacientes intervenidos de hernioplastia como CMA tienen un adecuado control del dolor considerado como EVA $\leq 3$ durante toda su estancia hospitalaria.

- Valorar si los pacientes consiguen un adecuado control del dolor en su domicilio con la pauta analgésica que el anestesista le adjunta al alta, es decir, si la pauta de analgesia es suficiente y eficaz para mantenerse en los márgenes de molestia y de dolor leve tras la cirugía a la que han sido sometidos (EVA $\leq 3)$.

\section{MATERIAL Y MÉTODOS}

\section{Diseño del estudio}

Se realiza un estudio observacional prospectivo, longitudinal y descriptivo para valorar el dolor postquirúrgico de los individuos intervenidos quirúrgicamente de hernioplastia y los analgésicos precisos para prevenir, cesar o disminuir el dolor.

Ámbito y período 
El estudio fue desarrollado en el Servicio de Anestesia y Reanimación del Hospital Universitario Santa $\mathrm{M}^{\mathrm{a}}$ del Rosell del Área II de Cartagena, durante los meses de octubre a diciembre de 2014.

\section{Participantes}

La población elegida para el estudio fueron individuos que acudían a dicho hospital para ser intervenidos quirúrgicamente de hernioplastia con alta hospitalaria ese mismo día desde el hospital de día quirúrgico (HDQ). En ese año se realizaron unas 400 intervenciones de hernioplastia en dicho hospital como CMA.

Los criterios de inclusión fueron todos los individuos mayores de edad que se intervinieran de hernioplastia según el sistema de clasificación American Society of Anesthesiologists (ASA) con riesgo anestésico ASA I-II, y III muy seleccionados. La selección de procesos fueron: hernias inguino-crurales (incluye las bilaterales y las recidivadas previa selección), hernias de pared abdominal (epigástricas, umbilicales, Spiegel) y eventraciones.

Los criterios de exclusión dentro de la muestra fueron individuos con discapacidad mental, demencias o que presentaran barrera idiomática.

Los individuos seleccionados fueron informados del estudio y dieron su autorización, firmando un consentimiento informado en la sala de premedicación antes de iniciar la intervención.

\section{Fuente y recogida de información}

El seguimiento del individuo ha sido realizado por una enfermera del Servicio de Anestesia y Reanimación que ha explicado el procedimiento, pasado el consentimiento, registrado los datos y llamado por teléfono.

Para la recogida de información se ha realizado un documento ajustado a nuestros objetivos (anexo I) y hemos utilizado varias fuentes de información:

- De la valoración anestésica realizada según el sistema de clasificación ASA, se han registrado datos como el sexo, la edad, si está en tratamiento en la unidad de dolor crónico (UDO),... En esta última categoría hemos incluido a los pacientes que toman analgésicos de forma habitual.

- De la hoja intraoperatoria, se ha registrado el tipo de hernioplastia que ha sido clasificado en hernia inguinal, hernia umbilical y hernia múltiple; el tipo de anestesia utilizado que se ha agrupado en anestesia general o sedación; si se infiltra o bloquea a nivel local la zona de la hernioplastia; y los analgésicos que se le administran de modo preventivo durante la intervención quirúrgica.

- Durante su estancia en reanimación, se realizaron varias valoraciones del dolor con la Escala Visual y Verbal Analógica (EVA) que llevaba incorporada la Escala Visual Numérica (EVN) y la Escala de Expresión Facial (Anexo II). Esta escala va desde 0 que es ningún tipo de dolor hasta 10 que es un dolor insoportable. Se registró el dolor al ingreso o cuando despertaban de la anestesia (a veces llegaban aún dormidos), cuando tenían un pico de dolor, a los 30-60 minutos en el caso de administrar algún analgésico de rescate y al alta. Actualmente se considera que el dolor postquirúrgico debe ser de 30 
menos en la escala numérica o visual analógica para juzgar como efectivo el tratamiento en reposo. ${ }^{15,18} \mathrm{La}$ escala del dolor EVA la hemos agrupado en nuestro estudio en: sin dolor o molestias (0-1), dolor leve (2-3), dolor moderado (4-7) y dolor intenso (8-10).

- Se han registrado los analgésicos administrados en reanimación, que se agruparon en 3 divisiones: el grupo de no opiáceos que incluye a los antiinflamatorios no esteroideos (AINES), paracetamol y metamizol, opioides menores y opioides mayores.

- Los datos valorados por vía telefónica fueron: EVA al alta en el CMA, dolor durante la noche y mañana siguiente a la intervención (destacando EVA mínimo y EVA máximo), y los analgésicos que los individuos se administran vía oral para controlar el dolor, según el protocolo de analgesia recibido por los anestesistas al alta. Este protocolo consta de 2 pautas de analgésicos, la cual depende de si es alérgico a AINES o no.

- Si no es alérgico a AINES (Anexo III): el individuo debe tomar dexketoprofeno $25 \mathrm{mg}$ vía oral cada 8 horas. Mientras dura el tratamiento con este medicamento hay que asociarle omeprazol $20 \mathrm{mg}$ vía oral cada 24 horas. Si el dolor no está controlado, hay que asociar paracetamol 1 gramo vía oral cada 8 horas alternándolos. Y si persiste el dolor con los analgésicos anteriores, se debe asociar tramadol retard $50 \mathrm{mg}$ vía oral 1 comprimido cada 12 horas, tras tomar domperidona 10 mg 1 hora antes del tramadol.

- Si es alérgico a AINES (Anexo IV): es la misma pauta mencionada pero no se administra dexketoprofeno. En su lugar, se administra metamizol 500 mg vía oral cada 8 horas.

\section{Análisis estadístico}

El análisis estadístico se realizó con el paquete estadístico SPSS para Windows versión 19.0. Se realizó un análisis descriptivo (frecuencias y medidas de tendencia central) de las variables relativas a las características de los sujetos y las variables relativas a los datos expresados de la escala visual y verbal del dolor (EVA) por los individuos de la muestra y de los analgésicos administrados.

\section{Legislación}

El estudio fue autorizado por la jefa de Servicio de Anestesia y Reanimación y aprobado por la Comisión de Investigación del Área II de Cartagena del Servicio Murciano de Salud.

\section{RESULTADOS}

\section{Características de los participantes}

Participaron 70 individuos intervenidos de hernioplastia en CMA, de los cuales, 57 fueron varones y 13 mujeres. Se tuvo una pérdida de información vía telefónica de un individuo porque no se le localizó; manteniendo la información obtenida en el hospital. 
La mayoría de la población a estudio fueron varones $(81,4 \%)$, menores de 60 años $(68,6 \%)$ e intervenidos de hernia inguinal $(64,3 \%)$. La anestesia que más se utilizó en las hernioplastias cumpliendo criterios de HDQ, fue la anestesia general en un $92,9 \%$. Y un $91,4 \%$ de los individuos no estaban tomando ningún analgésico de forma habitual o tratamiento crónico para aliviar el dolor. La media de edad es de 53,16 años con una desviación típica de 13,305. El 100\% de los pacientes fueron infiltrados a nivel local.

Tabla I. Características de los participantes

\begin{tabular}{|l|c|c|}
\hline \multicolumn{1}{|c|}{ VARIABLES } & $\mathbf{n}$ & PORCENTAJE \\
\hline Género & & \\
\hline Masculino & 57 & $81,4 \%$ \\
\hline Femenino & 13 & $18,6 \%$ \\
\hline Edad media:53,16 & & $68,6 \%$ \\
\hline$<60$ & 48 & $31,4 \%$ \\
\hline$>60$ & 22 & \\
\hline UDO & & $8,6 \%$ \\
\hline Sí & 6 & $91,4 \%$ \\
\hline No & 64 & $64,3 \%$ \\
\hline Intervención & & $28,6 \%$ \\
\hline Hernia inguinal & 45 & $7,1 \%$ \\
\hline Hernia umbilical & 20 & \\
\hline Hernia múltiple & 5 & $7,1 \%$ \\
\hline Anestesia & & $92,9 \%$ \\
\hline Sedación & 5 & \\
\hline General & 65 & \\
\hline
\end{tabular}

\section{Recogida de información de la escala EVA durante su estancia en reanimación, al alta del HDQ y en su domicilio por vía telefónica}

Al ingreso en reanimación, según la escala visual y verbal analógica del dolor, un $80 \%$ no tuvieron ningún dolor o tan sólo una molestia (EVA $\leq 1$ ), y un $14,3 \%$ tuvo un dolor leve (EVA $>1 \mathrm{y} \leq 3)$.

Sólo un 5,7\% de los individuos apreció su dolor como un dolor moderado. Durante su estancia en reanimación, los individuos que no tenían dolor y como mucho notaban una molestia fueron un 58,6\%; aquellos que tuvieron un dolor leve fueron el $34,3 \%$ de la muestra y sólo un $7,1 \%$ tuvieron un dolor de tipo moderado-intenso.

A la hora de dar el alta en reanimación, un 98,6\% de los individuos no tenían dolor o sólo referían tener molestias, y el 1,4\% restante presentaban un EVA: 3 , es decir, un dolor considerado leve.

La media de la EVA al ingreso en reanimación fue de $0,76( \pm 1,488)$, de la estancia fue de 0,50 $( \pm 0,676)$ y al alta fue de $0,63( \pm 0,569)$. 


\section{Gráfica 1 EVA al ingreso, durante su estancia y al alta de reanimación}

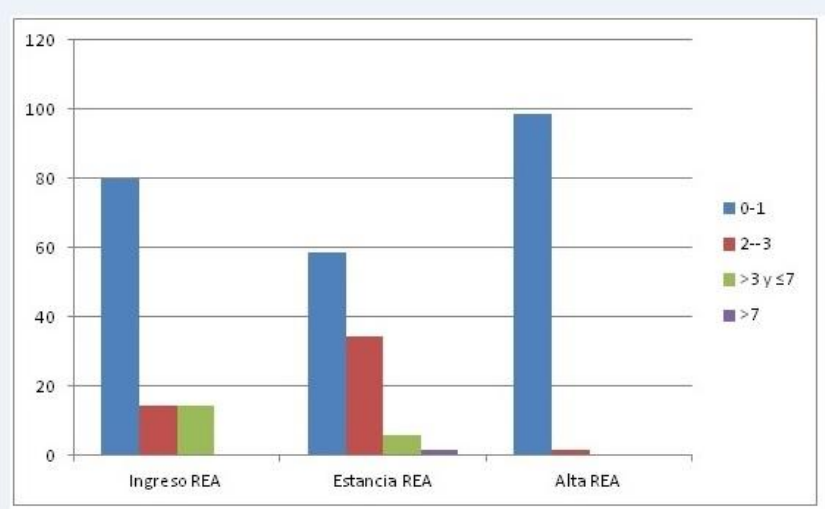

Un $82,6 \%$ de los individuos de la muestra se fueron a su domicilio sin dolor o sólo con molestias. Ningún paciente se fue de alta del HDQ con un dolor en la escala EVA > 4 . Siendo un $8,7 \%$ los individuos que tuvieron un dolor leve en movimiento, y sólo un 2,9\% los que percibieron un dolor moderado de EVA: 4. Por lo tanto podemos decir que la media de la EVA al alta del HDQ fue de 0,90 $( \pm 0,987)$.

Tabla II EVA al alta del CMA

\begin{tabular}{|l|r|r|r|}
\hline \multicolumn{1}{|c|}{ EVA } & Frecuencia & \multicolumn{1}{c|}{ Porcentaje } & \multicolumn{1}{c|}{ Porcentaje válido } \\
\hline 0 & 27 & 38,6 & 39,1 \\
\hline 1 & 30 & 42,9 & 43,5 \\
2 & 3 & 8,6 & 8,7 \\
\hline 3 & 6 & 5,7 & 5,8 \\
4 & 2 & 2,9 & 2,9 \\
Total & 69 & 98,6 & 100,0 \\
Perdido & 1 & 1,4 & \\
Total & 70 & 100,0 & \\
\hline
\end{tabular}

En su domicilio, vía telefónica, un $87 \%$ de los individuos refirieron no tener dolor o sentir sólo molestias. Un 11,5\% presentaban dolor leve refiriéndose a su dolor máximo. Y alrededor del $20 \%$ de los individuos estaban mal controlados, presentando un EVA $\geq 4$.

Tabla III. EVA máximo en su domicilio las primeras 24 horas

\begin{tabular}{|l|r|r|r|}
\hline \multicolumn{1}{|c|}{ EVA } & Frecuencia & \multicolumn{1}{c|}{ Porcentaje } & Porcentaje válido \\
\hline $\mathbf{0}$ & $\mathbf{1 1}$ & 15,7 & 15,9 \\
\hline $\mathbf{1}$ & $\mathbf{2 3}$ & 32,9 & 33,3 \\
\hline $\mathbf{2}$ & $\mathbf{1 1}$ & 15,7 & 15,9 \\
\hline $\mathbf{3}$ & $\mathbf{1 0}$ & 14,3 & 14,5 \\
\hline $\mathbf{4}$ & $\mathbf{3}$ & 4,3 & 4,3 \\
\hline $\mathbf{5}$ & $\mathbf{4}$ & 5,7 & 5,8 \\
\hline $\mathbf{6}$ & $\mathbf{4}$ & 5,7 & 5,8 \\
\hline $\mathbf{7}$ & $\mathbf{3}$ & 4,3 & 4,3 \\
\hline Total & 69 & 98,6 & 100,0 \\
\hline Perdidos & $\mathbf{1}$ & 1,4 & \\
\hline Total & $\mathbf{1 0}$ & 100,0 & \\
\hline
\end{tabular}


Sin embargo, sólo un 1,4\% presentó un dolor moderado como dolor mínimo, que suele coincidir con el dolor sufrido en reposo.

La media del dolor máximo domiciliario fue de 2,20 $( \pm 1,960)$, y la del dolor mínimo de $0,49( \pm 0,851)$.

Tabla IV. EVA mínimo en su domicilio las primeras 24 horas

\begin{tabular}{|l|r|r|r|}
\hline \multicolumn{1}{|c|}{ EVA } & Frecuencia & Porcentaje & Porcentaje válido \\
\hline 0 & 47 & 67,1 & 68,1 \\
\hline 1 & 13 & 18,6 & 18,8 \\
\hline 2 & 7 & 10,0 & 10,1 \\
\hline 3 & 1 & 1,4 & 1,4 \\
\hline 4 & 1 & 1,4 & 1,4 \\
\hline Total & 69 & 98,6 & 100,0 \\
Perdidos & 1 & 1,4 & \\
\hline Total & 70 & 100,0 & \\
\hline
\end{tabular}

Una vez valorada la EVA en varias ocasiones tras la intervención, se puede apreciar que un $37,1 \%$ no presentaron ningún dolor durante sus primeras 24 horas.

\section{Analgésicos administrados durante la intervención quirúrgica, en reanimación y en su domicilio}

El paracetamol 1 gramo vía intravenosa se utilizó como analgesia intraoperatoria en el $100 \%$ de los individuos. Siempre asociado a otro fármaco del grupo de los "no opiáceos", ya sea dexketoprofeno o metamizol. En un 11,4\% de los casos se añadió opiáceos al tratamiento analgésico $(5,7 \%$ con opioides menores y $5,7 \%$ con opioides mayores).

Tabla V. Analgésicos Intraoperatorios

\begin{tabular}{|l|c|c|}
\hline \multicolumn{1}{|c|}{ Analgésicos } & Frecuencia & Porcentaje \\
\hline No Opiodes & 62 & 88,6 \\
\hline No Opiodes + Opioides Menores & 4 & 5,7 \\
No Opiodes + Opioides Mayores & 4 & 5,7 \\
\hline Total & 70 & 100,0 \\
\hline
\end{tabular}

El dato más destacable de la tabla VI es que un $52,9 \%$ de los pacientes no precisaron ningún rescate analgésico durante su estancia en reanimación. También hemos querido resaltar que del $47,1 \%$ de los individuos restantes, sólo a un $15,7 \%$ se le administró morfina. 
Tabla VI. Rescate con analgésicos en reanimación

\begin{tabular}{|l|c|c|}
\hline \multicolumn{1}{|c|}{ Analgésicos } & Frecuencia & Porcentaje \\
\hline No & 37 & 52,9 \\
\hline AINE & 12 & 17,1 \\
\hline Opioide menor & 7 & 10,0 \\
\hline Opioide mayor & 4 & 5,7 \\
\hline AINE + Opiode menor & 3 & 4,3 \\
AINE + Opiode mayor & 5 & 7,1 \\
Varios rescates opioide & 2 & 2,9 \\
mayor & & \\
\hline Total & 70 & 100,0 \\
\hline
\end{tabular}

A pesar de tener un protocolo de analgesia domiciliaria facilitado por los anestesistas, mencionado anteriormente en el material y métodos, lo cierto es, que no hay relación directa entre el dolor y los analgésicos que los propios pacientes se administran vía oral.

\section{Tabla VII. Tabla de analgésicos administrados en domicilio}

\begin{tabular}{|l|r|r|r|}
\hline \multicolumn{1}{|c|}{ Analgésicos } & Frecuencia & Porcentaje & Porcentaje válido \\
\hline Desketoprofeno o Paracetamol & 17 & 24,3 & 24,6 \\
o metamizol & & & \\
\hline Metamizol + Paracetamol & 6 & 8,6 & 8,7 \\
\hline Desketoprofeno + Paracetamol & 36 & 51,4 & 52,2 \\
\hline Metamizol/dexketoprofeno + & 5 & 7,1 & 7,2 \\
Paracetamol + Tramadol & & & \\
ninguno & 5 & 7,1 & 7,2 \\
Total & 69 & 98,6 & 100,0 \\
Perdidos & 1 & 1,4 & \\
\hline Total & 70 & 100,0 & \\
\hline
\end{tabular}

Los individuos se tomaron en un $24,6 \%$ sólo un analgésico de la categoría de los "no Opiáceos"; un 60,9\% 2 analgésicos de la categoría de los "no Opiáceos", ya sea, metamizol más paracetamol, o dexketoprofeno más paracetamol. En los extremos tenemos un $7,2 \%$ de individuos que no tomaron ningún analgésico, y en el mismo porcentaje los que a los 2 analgésicos "no Opiáceos" le sumaron la administración del tramadol.

\section{DISCUSIÓN}

El Dolor Agudo Postquirúrgico (DAP) ha sido subestimado durante mucho tiempo, en detrimento del estado del paciente. ${ }^{13}$ Sin embargo, debe ser tratado adecuadamente con el fin de evitar molestias en la recuperación de éstos. Respecto a este punto, podemos señalar que, en una revisión realizada a nivel europeo en el año 2012 , se encontró que la evaluación del DAP aún se realizaba en menos del $50 \%$ de los casos. $^{15}$

El DAP debe ser manejado por el anestesista que tiene un conocimiento adecuado de la farmacocinética y farmacodinámica de los analgésicos, así como las vías del dolor y técnicas especializadas, todo ello a través de Unidades de Dolor Agudo (UDA). De 
esta manera, se mejora el confort, se favorece la incorporación a sus actividades e incluso se puede disminuir la morbimortalidad del paciente. ${ }^{13,}{ }^{16} \mathrm{El}$ empleo de analgésicos constituye el pilar fundamental para conseguir un adecuado control del dolor por lo que nuestro propósito fue conocer y evaluar el DAP, y el uso más frecuente de analgésicos. Para ello, es fundamental e imprescindible, hacer valoraciones del dolor mediante escalas como la EVA.

Diversas publicaciones han evidenciado que la asistencia del anestesista en la cirugía cuando ésta se realiza con anestesia local es imprescindible, ya que ofrece seguridad al paciente y al equipo de cirujanos. ${ }^{1}$ El manejo del dolor es una responsabilidad compartida, por lo que es imprescindible identificar las áreas de competencia de cada uno dentro del equipo asistencial. La valoración del dolor por parte de la enfermería es condición indispensable para un correcto manejo del mismo en el paciente sometido a intervención quirúrgica. En base a los resultados obtenidos mediante las escalas de valoración del dolor, el anestesista puede establecer el tratamiento más adecuado a las necesidades y características individuales de cada paciente. P. García et al. ${ }^{15}$ considera en 2005, según el documento "Dolor postoperatorio en España: primer documento de consenso", que se debe establecer un nivel máximo de dolor por debajo del cual se estime que existe un adecuado alivio del mismo, estableciendo este valor como aceptable en un nivel de 3 en la EVA. Según estos criterios, casi toda nuestra muestra tenía una analgesia adecuada durante las primeras 24 horas postquirúrgicas.

En el estudio de Hernández-Saldívar et al. ${ }^{13}$ más de la mitad de sus pacientes al ingreso en la unidad de cuidados postanestésicos presentaron ningún dolor o dolor leve (EVA: 0-4) y al alta, la mayoría cumplieron este criterio, con resultados similares a nuestros estudio.

Según una encuesta publicada en 2004 llevada a cabo por la Sociedad Española del Dolor (S.E.D.) sobre la incidencia del DAP en 11 hospitales de España, los resultados indican que el $68 \%$ de los pacientes presentaban dolor en la primera hora del postoperatorio. ${ }^{15}$ En nuestro estudio, en reanimación presentaron algún tipo de dolor $(E V A \geq 2)$ menos del $50 \%$ de la muestra.

En nuestro Servicio de Anestesia y Reanimación, el 82,6\% de nuestros pacientes fueron dados de alta a su domicilio sin dolor y presentaron como dolor máximo en su domicilio 24 horas después de la intervención, un 4,3\% de los individuos, dolor intenso; un 20,2\% dolor moderado; un 30,4\% dolor leve y un $49,2 \%$ tan solo dolor o molestia. Por otro lado, los resultados obtenidos sobre el dolor mínimo a las 24 horas de la intervención fue que un $86,9 \%$ no presentaron dolor, sólo un $11,5 \%$ presentaron dolor leve y un 1,4\% presentaron dolor moderado. En el estudio de Valentín López et al. ${ }^{23} 158$ pacientes rellenaron una encuesta anónima sobre el dolor postoperatorio de los servicios de Cirugía General y Vascular. Los resultados obtenidos, dentro de su clasificación de las intervenciones que causan dolor moderado donde incluyen las hernioplastias, fueron un dolor postoperatorio máximo a las 24 horas de leve en un $20,6 \%$, de moderado en un $30,2 \%$ y de intenso en un $11 \%$. Sólo un $12,7 \%$ no presentaron dolor (EVA: 0) frente a nuestro 49,2\% (EVA $\leq 1$ ) ya mencionado antes. En el estudio Gallego et al. ${ }^{24}$ con un total de 377 pacientes, un 96,9\% refirieron haber presentado dolor en las últimas 24 horas. Dentro de nuestros criterios de dolor, podemos decir que presentaron algún dolor un 62,8\% durante sus primeras 24 horas tras la intervención. 
Consideramos imprescindible estandarizar protocolos analgésicos de manejo institucional para los diferentes tipos de cirugías, con el fin de brindar más satisfacción a los pacientes. Es fundamental que el personal médico y de enfermería efectúe periódicamente una valoración del dolor percibido por el paciente, a efecto de adecuar la terapia analgésica en respuesta al dolor. Al igual que en el estudio Reyes Fierro, nuestro estudio demuestra que los pacientes se benefician con la analgesia multimodal a fin de mejorar el alivio del dolor y minimizar los efectos adversos. ${ }^{21,}{ }^{25} \mathrm{En}$ estudios recientes se ha comprobado la utilidad de este método con su empleo tanto preventivo como después de la cirugía, ya que reduce significativamente el dolor postoperatorio. $^{21}$

Los resultados obtenidos en nuestra encuesta nos indican que la analgesia pautada y prevista permitió un alivio adecuado del dolor con los movimientos, y por tanto, los pacientes pudieron retomar su movilidad habitual de manera temprana, recuperando así su ritmo de vida en el menor tiempo posible sin añadir mayores complicaciones. Dentro de la analgesia multimodal utilizada en nuestro estudio, queremos destacar que todos los pacientes fueron infiltrados con anestesia local y se les ha administrado paracetamol; de este último, se ha demostrado su eficacia en el tratamiento del dolor moderado postoperatorio y en otros muchos tipos de dolor agudo. ${ }^{20}$ Los AINES constituyen la base del manejo de dolor postoperatorio de los individuos intervenidos con cirugía ambulatoria, como ocurre en nuestro trabajo realizado, ya que el uso de opioides debe reservarse para aquellos casos de dolor severo o que no cesan con los analgésicos "no opioides". ${ }^{20},{ }^{21} \mathrm{En}$ el estudio realizado por Vázquez et al. ${ }^{17}$ la analgesia postoperatoria se maneja con AINES más frecuentemente, con un alto porcentaje de dolor aceptable, tal como ocurre en nuestro estudio.

De acuerdo a los resultados que hemos obtenido, y corroborado por numerosos estudios previos, ${ }^{3}, 6-7,12,14,15,20-21$ el uso de la anestesia local combinado con el protocolo analgésico establecido en el CMA, en este tipo de cirugía parece ser altamente recomendable, ya que se produce menos dolor en el periodo postoperatorio inmediato y buen manejo en el domicilio, disminuyendo las complicaciones atribuibles al DAP. En nuestro estudio, todos los individuos fueron infiltrados con anestesia local; unos junto a una sedación en algunas hernias umbilicales, y el resto combinado con anestesia general (mascarilla laríngea). También se puede destacar de nuestro estudio que la mayoría eran varones e intervenidos de hernia inguinal, coincidiendo con lo planteado en la literatura revisada. ${ }^{2,19} \mathrm{En}$ el estudio de Adelsdorfer et al. ${ }^{26}$ su predominio de hernioplastia en cirugía ambulatoria fue masculino $(90,7 \%)$ con un promedio de edad de 54,3 años (17-84), y en el nuestro, la media de edad fue de 53,16 años y un predominio de varones en un $81,4 \%$. López Rodríguez et al. ${ }^{27}$ obtuvieron resultados similares en su trabajo.

Respecto al uso de la anestesia general (mascarilla laríngea) comparado a la anestesia intradural, según el estudio de M. Fernández-Ordoñez et al. Se mostró una efectividad similar pero con una relación coste-efectividad mayor para la anestesia general. ${ }^{28}$ Sin embargo, en un estudio aleatorio y doble ciego comparando los tres tipos de anestesia en hernioplastia inguinal comentados anteriormente, Tverskox $\mathrm{M}$ et al. ${ }^{7}$ demostraron una disminución significativa del dolor postoperatorio con la anestesia local y espinal, comparado con la anestesia general, sin encontrar diferencia significativa entre los 2 primeros grupos. Asimismo, demostraron una prolongación en el tiempo transcurrido en el postoperatorio frente al requerimiento analgésico, muy superior en el grupo con anestesia local. Suarez G. et al. ${ }^{29}$ asocia la intervención de hernia inguinal mediante la técnica de PHS (prolene hernia system) 
realizada bajo anestesia local y sedación en cirugía ambulatoria a una morbilidad postoperatoria mínima, niveles de DAP controlables con medicación oral, movilización precoz y reducción de costes.

Entre las limitaciones de nuestro estudio, destacamos la falta de registro de la medicación que tiene efecto coadyuvante analgésico (corticoides), la medicación utilizada para la inducción (fentanilo), y los analgésicos administrados en la sala de CMA. Además, tenemos una modesta muestra con 70 participantes de los 400 anuales. A pesar de no obtener diferencias significativas entre los resultados, sí podemos afirmar que presenta relevancia clínica al conocer la calidad del protocolo analgésico dado al alta a nuestros participantes, que son satisfactorios con margen de mejora; y nuestra pequeña aportación dentro de los estudios de EVA en DAP en CMA ya existentes. Dentro del reto de realizar una buena valoración del dolor mediante escalas para minimizarlo o cesarlo, se nos abre una probable línea de investigación realizando una comparativa en este tipo de pacientes intervenidos de hernioplastia, utilizando la técnica de la infiltración local comparada con realizar un bloqueo en la zona a intervenir, y posteriormente, evaluar el dolor en dichos casos con varias escalas de valoración.

\section{CONCLUSIONES}

1. La mayoría de los individuos no tuvieron dolor o refirieron una molestia durante las primeras 24 horas tras la intervención quirúrgica, a excepción de la estancia en reanimación que sólo la mitad de la muestra refirió un EVA $\leq 1$. Los individuos restantes que sí presentaron dolor en su mayoría fue leve.

2. Casi todos los individuos intervenidos de hernioplastia vía CMA presentaron un adecuado control del dolor postoperatorio.

3. Se realizó un abordaje multimodal:

- A todos los individuos se les administró analgésicos intraoperatorios, principalmente no opiáceos, y se les infiltró con anestésico local.

- En reanimación, los analgésicos más usados fueron los no opiáceos seguido de los opiáceos mayores (morfina).

- En su domicilio, más de la mitad de la muestra tomó 2 analgésicos no opiáceos.

4. Se considera eficaz la pauta de analgesia domiciliaria indicada por los anestesistas al alta, consiguiéndose un adecuado control del dolor.

\section{REFERENCIAS}

1. Ibar, Ricardo Sergio Hernández, et al. Anestesia local para el tratamiento de la hernia inguinal primaria. Cirujano General, 2007, vol. 29, no 3-2007, p. 188-191. http://www.medigraphic.com/pdfs/cirgen/cg-2007/cg073c.pdf

2., Leonardo L. Perera R. Inguinal hernia. Ambulatory traetment versus hospitalized. Mediciego. 2012, Vol 18, Supl.2.

3. Martínez Ramos C. Tratamiento de la hernia inguinal mediante cirugía mayor ambulatoria. Reduca. 2009; Vol 1, No.1. 
4. De Hernia, Asociación Mexicana. Guías de Práctica Clínica para Hernias de la Pared Abdominal. Asociación Mexicana de Hernia [en línea], 2009, p. 23-41.

5. Cruz Rodríguez, J, et al. Cirugía mayor ambulatoria y de corta estadía en el tratamiento del paciente con hernia abdominal externa. Revista Habanera de Ciencias Médicas, 2014, Vol 13, No 6, p. 893-901.

6. Acevedo A, Gallego, A. Cirugía mayor ambulatoria (CMA) de las hernias. Experiencia de 5 años en el CRS Cordillera Oriente de la ciudad de Santiago. Rev Chil Cir, 2004, Vol 56, p. 166-171.

7. De la Cuadra R, et al. Anestesia local vs anestesia espinal en hernioplastia inguinal: comparación de efecto anestésico postoperatorio. Rev chil cir, 2002, Vol 54, No 6, p. 649-53.

8. Acevedo A, Lombardi J. Cirugía de las hernias con anestesia local. Rev Chil Cir, 2003, Vol 55, p. 520-526.

9. Whizar-Lugo VM, Martínez NG, Torres, JCH. Polémicas en anestesia subaracnoidea. Anestesia en México, 2004, Vol 16, No 2.

10. Peralta-Zamora E. Actualidades y nuevas perspectivas de la anestesia neuroaxial. Rev Mex Anest, 2007, Vol 30, p. S256-S260.

11. Dálbora J, et al. Factores de riesgo de retención urinaria aguda en cirugía mayor ambulatoria bajo anestesia espinal. Revista chilena de cirugía, 2008, Vol 60, No 2, p. 139-144.

12. López-Portero S, et al. El dolor agudo postquirúrgico en el adulto" la 5a constante". 2014. URL

https://uvadoc.uva.es/handle/10324/5936.

13. Hernández-Zaldivar ML. Manejo del dolor postoperatorio: Experiencia terapéutica en Unidad de Terapia Quirúrgica Central del Hospital General de México. Rev Mexicana de Anestesiología, 2008, Vol 31, No 1, p. 246-251.

14. De La Cala F. Dolor postoperatorio: analgesia multimodal. Patología del aparato locomotor, 2004, Vol 2, No 3, p. 176-188.

15. Rojo $A$, et al. Manejo del dolor. Eficacia de las técnicas de valoración de las pautas analgésicas en pacientes sometidos a cirugía. 2014. URL disponible en: http://uvadoc.uva.es/handle/10324/5633

16. Vidal MA, et al. Estudio observacional sobre el dolor postoperatorio leve o moderado desde el punto de vista del anestesiólogo en España. PATHOS. Rev Soc Esp Dolor, 2007, Vol.8, p. 550-67.

17. Vásquez MC. Manejo del dolor postquirúrgico en los pacientes del servicio de cirugía y gineco-obstetricia del Hospital Homero Castanier Crespo de la ciudad de Azogues, durante el año 2011. 2012. URL disponible en: http://dspace.ucuenca.edu.ec/handle/123456789/3421

18. Martínez-Bisbal AL, Rodríguez NT. Dolor postoperatorio: enfoque procedimientoespecífico. Revista Ciencias Biomédicas, 2012, Vol. 3, No 2, p. 360- 372.

19. Muñoz J. M. Manual de dolor agudo postoperatorio. Madrid: Ergon; 2010

20. Bujedo BM, et al. Estrategias para el abordaje multimodal del dolor y de la recuperación postoperatoria. Rev. Esp. Anestesiol. Reanim, 2007, Vol. 54, No 1, p. 29- 40.

21. Mejía S, Sánchez Y. Recomendaciones prácticas de manejo anestésico para cirugía ambulatoria. URL: http://www.anestesianet.com/unal/ambulatorio.htm

22. Alonso AL, Ardizone I. La Escala Visual Analógica. 2013, p. 2-43

23. López, B. Valentín, et al. Atención del dolor postoperatorio en un hospital de tercer nivel: situación inicial previa a la implantación de un programa de calidad. Rev. Esp. Anestesiol. Reanim, 2006, vol. 53, p. 408-418. 
24. Gallego, J. I., et al. Estimación de la prevalencia e intensidad del dolor postoperatorio y su relación con la satisfacción de los pacientes. Rev Soc Esp Dolor, 2004, vol. 11, p. 197-202.

25. Reyes Fierro A. et al. Postoperative pain: multimodal analgesia. Patología del aparato locomotor 2004; 2 (3); p.176-188.

26. Complicaciones postoperatorias de la serie prospectiva de pacientes con hernioplastia inguinal, en protocolo de hospitalización acortada del Hospital Dr. Gustavo Fricke de Viña del Mar. Revista chilena de cirugía, 2007, vol. 59, no 6, p. 436-442

27. López Rodríguez, Pedro Rolando, et al. Nuestra experiencia de diez años en la herniorrafia de Mohan P. Desarda. Revista Cubana de Cirugía, 2013, vol. 52, no 2, p. 118-125.

28. Fernández-Ordoñez M., Tenías J.M., Picazo-Yeste J. Anestesia subaracnoidea y anestesia general en el tratamiento quirúrgico de la hernia inguinal en pacientes ambulatorios. Análisis coste-efectividad. Revista Española de Anestesiología y Reanimación. 2015. Vol 62 № 02

29. Suarez $\mathrm{G}$ et al. Hernioplastia inguinal ambulatorio con anestesia local y sedación: análisis de 1840 pacientes tras 6 años de seguimiento. Cir May Amb. 2014. Vol 19, № 1: 3-6 


\section{ANEXOS}

Anexo l. Características del paciente.

Anexol. Características del paciente.

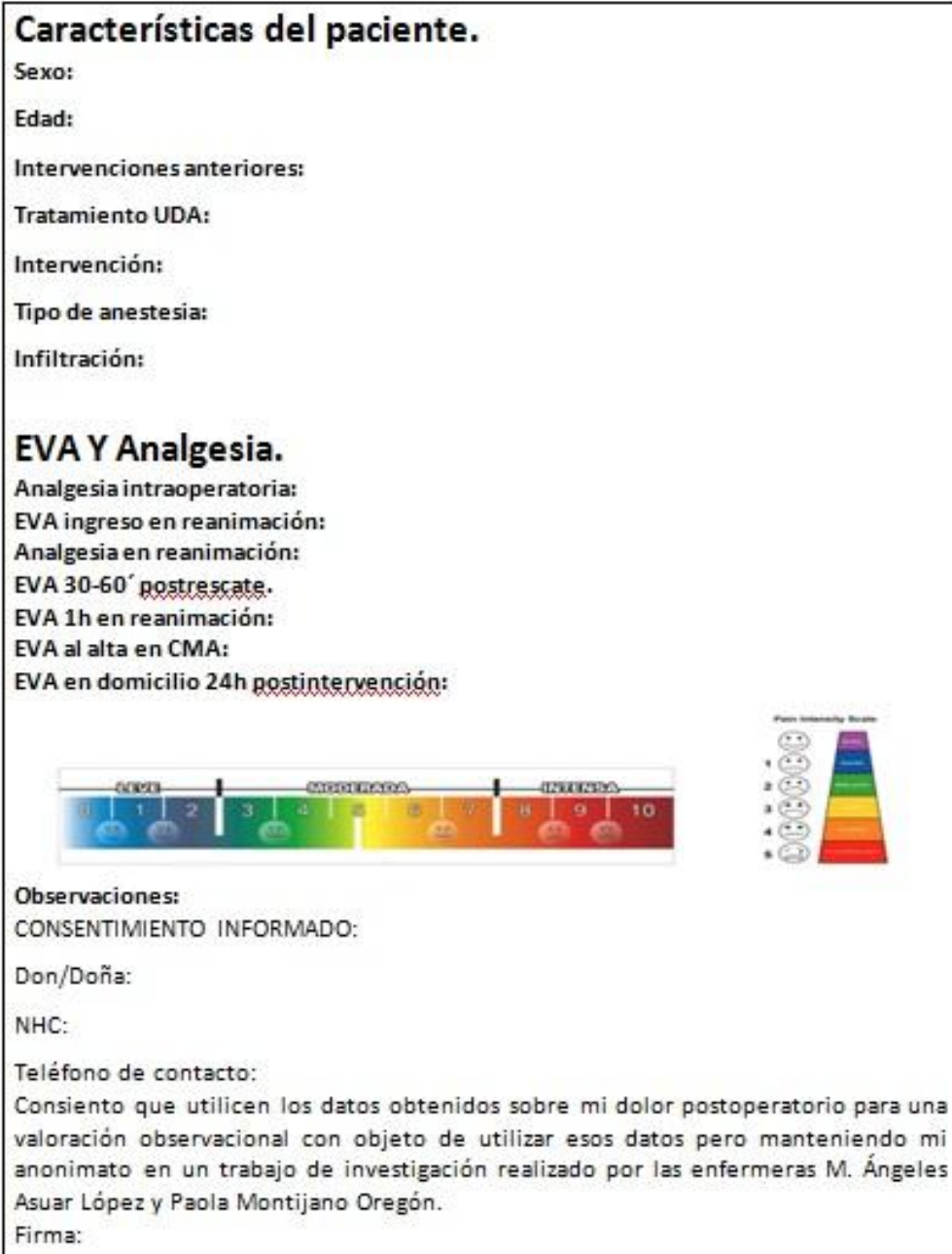

\section{[Anexo II. EVA.]}

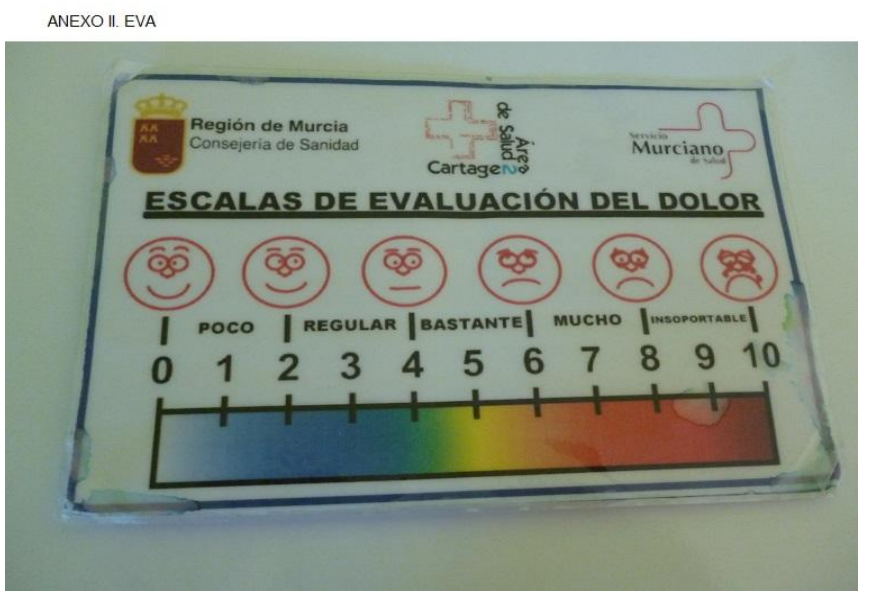


[Anexo III. Protocolo de analgesia domiciliaria.]

Región de Murcia

Conscjoria de Sanidad

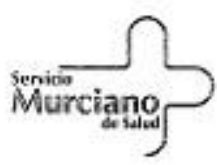

ANALGESIA DOMICILIARIA EN CMA Y CIRUGIA MENOR

- Dexketoprofeno $25 \mathrm{mg}$ (Enantyum 1 sobre granulado) vía oral cada 8 horas.

- Mientras dure el tratamiento analgésico con Enantyum ${ }^{*}$ hay que asociar

Omeprazol $20 \mathrm{mg}$ via oral cada 24 horas.

- Si dolor no controlado:

Asociar Paracetamol 1g via oral 1 comprimido cada 8 horas.

- Si persiste el dolor habiendo asociado el paracetamol:

Asociar Tramadol RETARD $50 \mathrm{mg}$ via oral 1 comprimido cada12 horas.

- Hay que tomar Domperidona $10 \mathrm{mg}$ (Motilium" 1 comp.) 1 hora antes de cada toma de Tramadol. 
[Anexo IV. Protocolo de analgesia domiciliaria para alérgicos a AINES.]

ANALGESIA DOMICILIARIA EN CMA Y CIRUGÍA MENOR.

\section{ALÉRGICOS A AINES}

- Metamizol $500 \mathrm{mg}$ (Nolotil 2 cápsulas) vía oral cada 8 horas.

- \$i dolor no controlado:

Asociar Paracetamol 1g via oral 1 comprimido cada 8 horas.

- Si persiste el dolor hablendo asociado el paracetamol:

Asociar Tramadol RETARD $50 \mathrm{mg}$ vía oral 1 comprimido cada 12 horas.

- Hay que tomar Domperidona $10 \mathrm{mg}$ (Motillum 1 comp.) 1 hora antes de cada toma de Tramadol.

Anestesiólogo/a:

Firma:

Recibido: 1 de junio 2015;

Aceptado: 22 de julio 2015

ISSN 1695-6141 